\title{
Observation and Analysis of Affinity Law Deviations through Tested Performance of Liquefied Gas Reaction Turbines
}

\author{
Sarah D. Alison-Youel \\ Research and Development, Cryodynamics Division, Ebara International Corporation, 350 Salomon Circle, Sparks, NV 89434, USA
}

Correspondence should be addressed to Sarah D. Alison-Youel, salison-youel@ebaraintl.com

Received 9 April 2008; Revised 14 August 2008; Accepted 11 October 2008

Recommended by Norihiko Iki

Liquefied gas reaction turbines are subject to the hydraulic affinity laws. Particularly for liquefied hydrocarbon gas-driven turbines, deviations from the affinity laws are encountered. In the case of reaction turbines, where the geometry is fixed, the affinity law relationships between flow, head, and rotational speed are relevant. Field experience confirms that the affinity law relationships are adequate, but that the predictions made also tend to deviate from real turbine performance. Part of the deviations seen may be attributed to the nonideal fluid; however, further examination is warranted. This paper presents an investigation into the affinity law relationships between head, flow, and rotational speed in conjunction with actual turbine performance. The three basic affinity law relationships are combined to form the most general performance equation. This equation subsequently incorporates both the affinity law relationships and the conservation of energy principal. Application of real turbine test data shows that this general performance equation presents a more accurate representation of turbine performance than the affinity law relationships alone.

Copyright (c) 2008 Sarah D. Alison-Youel. This is an open access article distributed under the Creative Commons Attribution License, which permits unrestricted use, distribution, and reproduction in any medium, provided the original work is properly cited.

\section{INTRODUCTION}

The liquefied gas reaction turbine industry began in the mid 1990s. The design and performance of liquefied hydrocarbon gas reaction turbines are extensively described in US patent 5659205 [1] and by Habets and Kimmel [2]. Lobanoff and Robert [3] stated that reaction turbines are pumps running in reverse; therefore, the affinity laws are applicable to the reaction-type turbines. In 1996, Kimmel [4] presented a turbine performance equation that incorporated the affinity laws and the energy of conservation. The intent of the current investigation is to find the most general polynomial that satisfies both the affinity law relationships for fixed geometry reaction turbines and the conservation of energy.

Relationships provided by the affinity laws and the conservation of energy are formed into a general polynomial resulting in a general performance equation. This equation is the most universal equation for constant geometry that incorporates both the affinity law relationships and the conservation of energy law.

The developed general performance equation is evaluated and analyzed using tested performance data from an
Ebara International Corporation (EIC) (NV, USA) liquefied gas reaction turbine. The results show an improvement in performance representation by the developed equation, as well as a probable relationship to pump performance.

\section{GENERAL PERFORMANCE EQUATION}

The affinity laws following relationship between the flow and rotational speeds at two operating points, $a$ and $b$,

$$
\frac{Q_{a}}{Q_{b}}=\frac{N_{a}}{N_{b}}=X,
$$

where $X$ is some constant. Again, at two operating points, $a$ and $b$, the affinity law relates the head, flow, and speed as follows:

$$
\frac{H_{a}}{H_{b}}=\frac{Q_{a}^{2}}{Q_{b}^{2}}=\frac{N_{a}^{2}}{N_{b}^{2}}=Y,
$$

where $Y$ is some constant [1]. If (1) is squared, then $Y$ must be equal to $X^{2}$, yielding the following relationships between 
TABLE 1: Values of $m$ and $n$.

\begin{tabular}{lll}
\hline$m$ & $n$ & $m+n=2$ \\
\hline 2 & 0 & $2+0=2$ \\
1 & 1 & $1+1=2$ \\
0 & 2 & $0+2=2$ \\
\hline
\end{tabular}

the two operating points:

$$
\begin{aligned}
Q_{a} & =X \cdot Q_{b}, \\
N_{a} & =X \cdot N_{b}, \\
H_{a} & =X^{2} \cdot H_{b} .
\end{aligned}
$$

In fluid-driven machinery, the head is a function of the flow and rotational speed, $H=f(Q, N)$. This function of flow and rotational speed is continuous; therefore, it can be developed into a Taylor series of two variables [5]:

$$
H=\sum_{m, n} c_{m n} Q^{m} N^{n}
$$

where $m$ and $n$ are nonnegative integers. When (4) is expanded, it takes the form

$$
\begin{aligned}
H= & c_{00}+c_{10} Q+c_{01} N+c_{20} Q^{2}+c_{02} N^{2}+c_{11} Q N \\
& +c_{30} Q^{3}+c_{03} N^{3}+c_{21} Q^{2} N+c_{12} Q N^{2}+\cdots+c_{m n} Q^{m} N^{n} .
\end{aligned}
$$

Using (4), the head at one operating point, $a$, is then

$$
H_{a}=\sum_{m, n} c_{m n} Q_{a}^{m} N_{a}^{n}
$$

Substituting the relationships shown in (3) into (6), a relationship is found for the operating point $b$ :

$$
X^{2} H_{b}=\sum_{m, n} c_{m n}\left(X Q_{b}\right)^{m}\left(X N_{b}\right)^{n} .
$$

Collecting terms and factoring out constants, the following results:

$$
X^{2} H_{b}=X^{m+n} \sum_{m, n} c_{m n} Q_{b}^{m} N_{b}^{n}
$$

To relate this general function to the affinity laws, $X^{2}$ must equal $X^{m+n}$, which leads to the constraint relationship $m+$ $n=2$. Since $m$ and $n$ must be positive integers, there are only three cases for values of $m$ and $n$ that make a true statement. The values for the three cases are shown in Table 1 .

To take (8) and make it specific to the affinity law, the values of $m$ and $n$ in Table 1 are substituted into (5), yielding

$$
H=c_{20} Q^{2}+c_{02} N^{2}+c_{11} Q N .
$$

Written more simply, substituting $\alpha, \beta$, and $\gamma$ for $c_{20}, c_{02}$, and $c_{11}$, respectively, a general performance equation is found

$$
H=\alpha Q^{2}+\beta N^{2}+\gamma Q N .
$$

The above equation is the most complete form of the energy equation that also follows the affinity laws. The head is the total static energy and it must equal the total kinetic energy. The traditional performance equation proposed by Kimmel in 1996 [4] (see (21) below), does not include all the terms for kinetic energy, whereas the general performance equation does. The constants, $\alpha, \beta$, and $\gamma$ are specific to an individual turbine.

\section{GENERAL PERFORMANCE EQUATION AND THE NO-LOAD CONDITION}

Equation (10) governs the performance of the turbine; therefore, it should also describe the no-load condition. In 1997, Kimmel [6] stated that under the no-load condition the inlet and outlet momentums must be equal, leading to the following relationship:

$$
Q=\lambda N
$$

Inserting this relationship into (10) and then simplifying, the following relationship is produced:

$$
H=\delta Q^{2},
$$

where,

$$
\delta=\alpha+\frac{\beta}{\lambda^{2}}+\frac{\gamma}{\lambda}
$$

Equation (10) coupled with the offshoot equation for noload operation, (12), provides general performance equations that follow the affinity law, conservation of energy, and momentum allowing for complete and superior representation of turbine performance.

\section{TURBINE DATA ANALYSIS}

Test data from EIC turbine model LX14-07 was used in the analysis and application of the general performance equation. Model LX14-07 is a single-phase liquefied gas reaction turbine designed for a nominal flow of $1400 \mathrm{~m}^{3} / \mathrm{h}$ and a nominal head of $700 \mathrm{~m}$.

During performance testing of the turbine, performance parameters relating to flow and head were recorded in addition to the rotational speed. The turbine flow parameter is recorded by measuring the pressure drop across a Venturi flowmeter. Flow is proportional to the square of the pressure resulting in flow parameter tested data in units of $\mathrm{kPa}^{1 / 2}$. The turbine head parameter is recorded by measuring the pressure drop across the turbine. Head is directly proportional to the differential pressure resulting in head parameter tested data in units of $\mathrm{kPa}$. Typically turbine flow and head are then calculated using the measured flow and head parameter data; however, in the analysis of the general performance equation, all calculations were made using the flow and head parameter tested data points and not the typically calculated head and flow. This was done in order to eliminate calculation error and to empirically evaluate the general performance equation. The turbine speed is measured by 
accelerometers and is recorded in revolutions per minute which is easily converted to revolutions per second for use in the analysis of the equation. The flow and head parameter data was recorded during the no-load condition and for three rotational speeds, $2400 \mathrm{RPM}, 3110 \mathrm{RPM}$, and $4000 \mathrm{RPM}$. For this particular test, at the speed of 3110 RPM the data was only recorded for a range approximately between flow parameters 3.71 and 4.25 . This does not greatly affect the results as a surface fit is applied to all the speed data.

A three-dimensional surface fit using the general performance equation and the tested data was performed to determine the values of $\alpha, \beta$, and $\delta$ in (10) specific to the tested data. It is assumed from the theory explained above that (10) was the best fit for the data. It follows, if a certain test point, $i$, is plugged into this equation, that there is an error associated, $E_{i}$, if the test point does not lie exactly on the surface generated by (10):

$$
E_{i}=\alpha Q_{i}^{2}+\beta N_{i}^{2}+\gamma Q_{i} N_{i}-H_{i}
$$

Some of these $E_{i}$ values are positive and some are negative. To eliminate the possibility of positive and negative error values canceling out, each $E_{i}$ is squared; this is called the square error. The square error for each data point is then summed as follows:

$$
\sum_{i} E_{i}^{2}=\sum_{i}\left(\alpha Q_{i}^{2}+\beta N_{i}^{2}+\gamma Q_{i} N_{i}-H_{i}\right)^{2} .
$$

To make a best fit, this square error sum should be a minimum. The minimum condition is found when the partial derivatives relative to $\alpha, \beta$, and $\gamma$ are found and set equal to zero. This provides three linear equations that are solved for the constants $\alpha, \beta$, and $\gamma$ for the data set. The following three linear equations are the result of the partial derivatives set to zero to find the constants $\alpha, \beta$, and $\gamma$ :

$$
\begin{aligned}
& \alpha=\frac{s_{2}^{2} s_{5}-s_{3} s_{5} s_{7}+s_{1} s_{7}^{2}+s_{3} s_{6} s_{8}-s_{2}\left(s_{4} s_{5}+s_{7} s_{8}\right)}{s_{2}^{3}+s_{3}^{2} s_{6}+s_{4} s_{7}^{2}-s_{2}\left(s_{4} s_{6}+2 s_{3} s_{7}\right)}, \\
& \beta=\frac{s_{3}^{2} s_{5}+s_{1}\left(s_{2}^{2}-s_{3} s_{7}\right)+s_{4} s_{7} s_{8}-s_{2}\left(s_{4} s_{5}+s_{3} s_{8}\right)}{s_{2}^{3}+s_{3}^{2} s_{6}+s_{4} s_{7}^{2}-s_{2}\left(s_{4} s_{6}+2 s_{3} s_{7}\right)}, \\
& \gamma=\frac{s_{1} s_{3} s_{6}+s_{4} s_{5} s_{7}-s_{2}\left(s_{3} s_{5}+s_{1} s_{7}\right)+s_{2}^{2} s_{8}-s_{4} s_{6} s_{8}}{s_{2}^{3}+s_{3}^{2} s_{6}+s_{4} s_{7}^{2}-s_{2}\left(s_{4} s_{6}+2 s_{3} s_{7}\right)} .
\end{aligned}
$$

Application of the test points to (16)-(18) yielded $\alpha=$ $8.088724, \beta=0.0886066$, and $\gamma=-0.2323394$, resulting in a general performance equation specific to the tested data:

$$
H=8.088724 Q^{2}+0.0886066 N^{2}-0.2323394 Q N .
$$

The negative sign associated with the third term was expected. The negative sign of third term is explained by the leakage flow and recirculation [7]. The results of this surface fit are plotted two-dimensionally by holding the rotational speed constant and are shown with corresponding tested flow and head parameter data points in Figure 1.

Application of the same square error method to (11), for the no-load condition, yields a relationship between $\lambda$ and

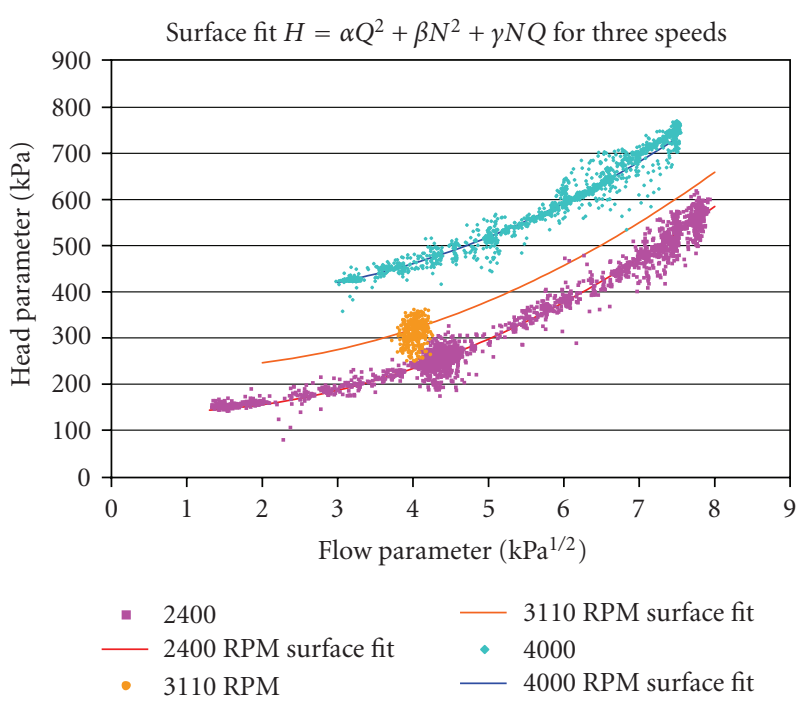

FIGURE 1: Head parameter versus flow parameter tested data with general performance equation surface fit.

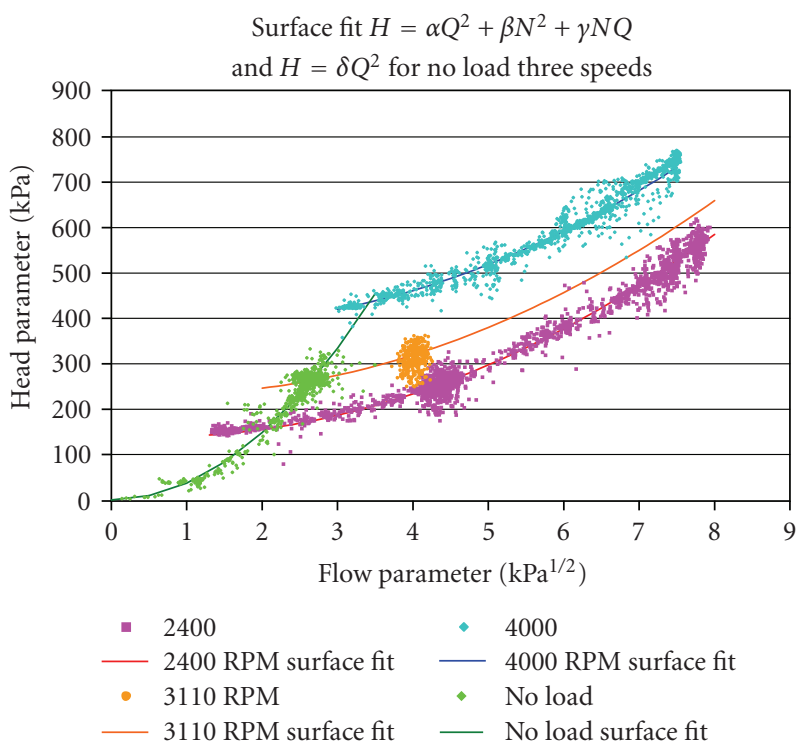

FIGURE 2: Head parameter versus flow parameter tested data with general performance equation surface fit including no load.

the tested data as follows:

$$
\lambda=\frac{\sum_{i} Q_{i} N_{i}}{\sum_{i} N_{i}^{2}} .
$$

The value of $\lambda$ was found to be 0.051374 . Substituted this into (13), along with $\alpha, \beta$, and $\gamma$, yielded the value, $\delta=$ 37.13874. This generated a surface fit for the no-load condition. Using the now known values for $\alpha, \beta, \gamma, \lambda$, and $\delta$, the surface fit for the no-load curve was to be added to the constant speed curves from Figure 1 and included the tested flow and head parameter data to show complete turbine performance envelope (see Figure 2). 


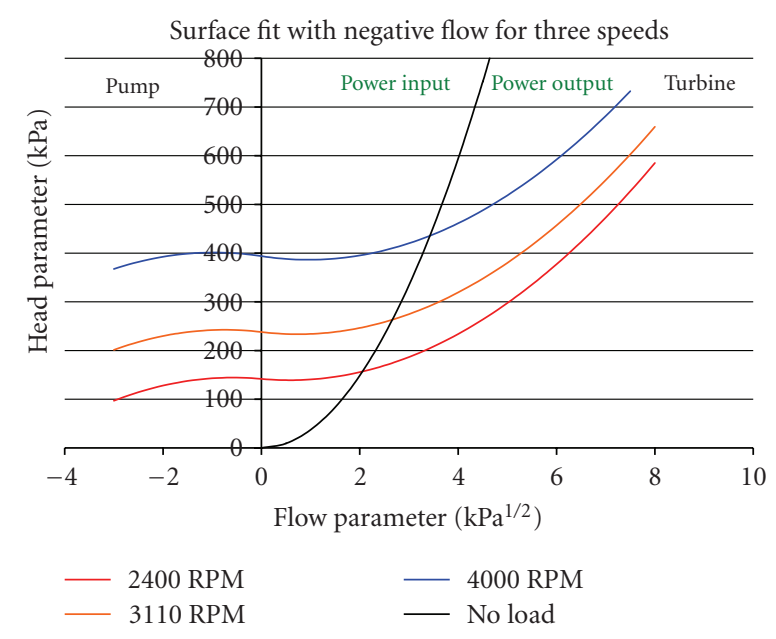

FIGURE 3: General performance equation for pumps and turbines.

The results from the three speeds and the no-load curve clearly show the applicability of the general performance equation. Furthermore, it can be shown that this equation is also applicable to pumps. Pumps also follow the affinity law relationships and conservation of energy principal; therefore, the general performance equation must also be applicable to pumps. To model this, the principal that a pump is a turbine running in reverse is employed. Essentially, a turbine running in reverse is just a turbine with negative flow. To show this, a range of flow and head parameter values for the same three speeds using (19) was plotted in Figure 3. As the flow parameter becomes negative, the curves in Figure 3 show a similarity to pump performance curves, supporting the applicability of (10) to pumps as well as turbines. These curves show a striking similarity to those presented by Stepanoff [7].

\section{IMPROVEMENT}

Figures 1 and 2 show that the general performance equation is an excellent fit to the tested data. To show actual improvement by (10), the same method and the tested data were applied to the traditional performance equation proposed by Kimmel in 1996 (see (21)) and then compared to results obtained by the general performance equation:

$$
H=A Q^{2}+B N^{2} .
$$

The sum of the square error for (21) is then as follows:

$$
\sum_{i} e_{i}^{2}=\sum_{i}\left(A Q_{i}^{2}+B N_{i}^{2}-H_{i}\right)^{2}
$$

In this case, there are only two constants to solve for, $A$ and $B$; therefore, there are only two partial derivatives of the sum of the square error to set to zero resulting in two linear equations as follows:

$$
\begin{aligned}
& A=\frac{s_{2} s_{5}-s_{1} s_{6}}{s_{2}^{2}-s_{4} s_{6}}, \\
& B=\frac{s_{1} s_{6}-s_{4} s_{5}}{s_{2}^{2}-s_{4} s_{6}} .
\end{aligned}
$$

Application of the tested data points to (23) and (24) yielded $A=7.120981$, and $B=0.0768359$, resulting in

$$
H=7.120981 Q^{2}+0.0768359 N^{2} .
$$

Now with (19) and (25), again the error was employed to check how well each equation fits the tested data. The square error was calculated for each method and then a ratio was taken to indicate any improvement. The ratio of the square error is then as follows:

$$
\text { Ratio }=\frac{\sum_{i} e_{i}^{2}}{\sum_{i} E_{i}^{2}} .
$$

The ratio of the square error was calculated to be 1.10 and the root mean-square error is then the square root of this ratio. This gives a root mean-square error of 1.05, indicating that the general performance equation is 1.05 times better than the traditional equation. These results show considerable improvement without a significantly more complicated equation.

\section{CONCLUSIONS}

A new general performance equation relating flow, head, and rotational speed for liquefied gas reaction turbines was developed and presented with performance test data. The general performance equation is the most general and complete equation for the performance of liquefied gas reaction turbines with constant geometry. In addition, from the analysis presented, there are several strong findings supporting the claim that the general performance equation is an improvement over the traditional performance equation presented by Kimmel [4]. The general performance employs the affinity law relationships between flow, head, and rotational speed with more accuracy. The third term in the general performance equation incorporates the experienced negative slope in a turbine performance curve. Furthermore, the general performance equation is also applicable to pumps. The strongest evidence supporting the improvement over the known affinity law is found in the error analysis. The error generated by the general performance equation is considerably less, ultimately providing a better engineering tool for the representation of both turbine and pump performances.

\section{NOMENCLATURE}

Q: $\quad$ Flow

$N$ : $\quad$ Rotational speed

$H: \quad$ Head

$a, b: \quad$ Distinct operating points

$X, Y$ : Unknown constants

$c: \quad$ Summation constant

$m, n$ : Summation indices

$\alpha, \beta, \gamma$ : General affinity law equation constants

$\lambda$ : $\quad$ Constant relating $Q$ and $N$ under no load

$\delta$ : $\quad$ Constant relating $H$ and $Q$ under no load

I: $\quad$ Summation index indicating a data point

$E$ : $\quad$ Error related to general performance equation 
$A, B$ : Affinity law and energy equation constants

$\lambda$ : $\quad$ Constant relating $Q$ and $N$ under no load

$\delta$ : $\quad$ Constant relating $H$ and $Q$ under no load

$I$ : Summation index indicating a data point

$E$ : $\quad$ Error related to general performance equation

$A, B$ : Affinity law and energy equation constants

$e$ : $\quad$ Error related to the affinity law and energy equations

$\begin{aligned} s_{1} & =\sum_{i} Q_{i}^{2} H_{i}, \\ s_{2} & =\sum_{i} N_{i}^{2} Q_{i}^{2}, \\ s_{3} & =\sum_{i} N_{i} Q_{i}^{3}, \\ s_{4} & =\sum_{i} Q_{i}^{4}, \\ s_{5} & =\sum_{i} H_{i} N_{i}^{2}, \\ s_{6} & =\sum_{i} N_{i}^{4}, \\ s_{7} & =\sum_{i} Q_{i} N_{i}^{3}, \\ s_{8} & =\sum_{i} H_{i} N_{i} Q_{i} .\end{aligned}$

\section{REFERENCES}

[1] G. L. Weisser, "Hydraulic turbine power generator incorporating axial thrust equalizing means," U.S. patent no. 5659205, 1997.

[2] G. L. G. M. Habets and H. E. Kimmel, "Development of hydraulic turbine in liquefied natural gas," in Proceedings of the 7th European Congress on Fluid Machinery for the Oil, Petrochemical, and Related Industries (IMechE '99), The Hague, The Netherlands, April 1999.

[3] V. S. Lobanoff and R. R. Robert, Centrifugal Pumps: Design \& Application, Gulf, Houston, Tex, USA, 2nd edition, 1992.

[4] H. E. Kimmel, "Variable speed turbine generators in LNG liquefaction plants," in Proceedings of the International Conference and Exhibition for The LNG, LPG and Natural Gas Industries (GASTECH '96), pp. 1-21, Vienna, Austria, December 1996.

[5] R. Courant and F. John, Introduction to Calculus and Analysis, Springer, New York, NY, USA, 2000.

[6] H. E. Kimmel, "Speed controlled turbine expanders," Hydrocarbon Engineering, May-June 1997.

[7] A. J. Stepanoff, Centrifugal and Axial Flow Pumps, John Wiley \& Sons, New York, NY, USA, 1957. 

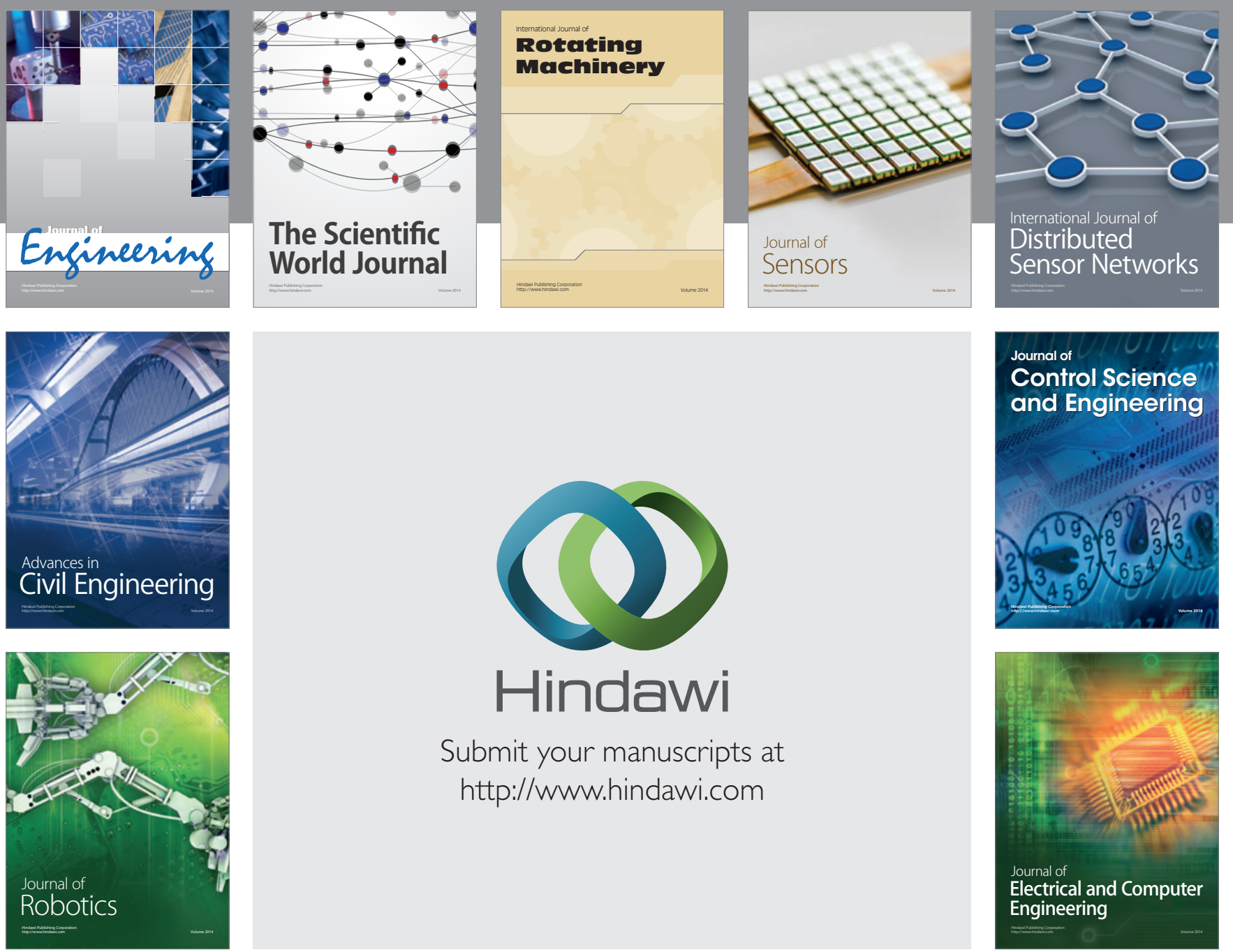

Submit your manuscripts at

http://www.hindawi.com
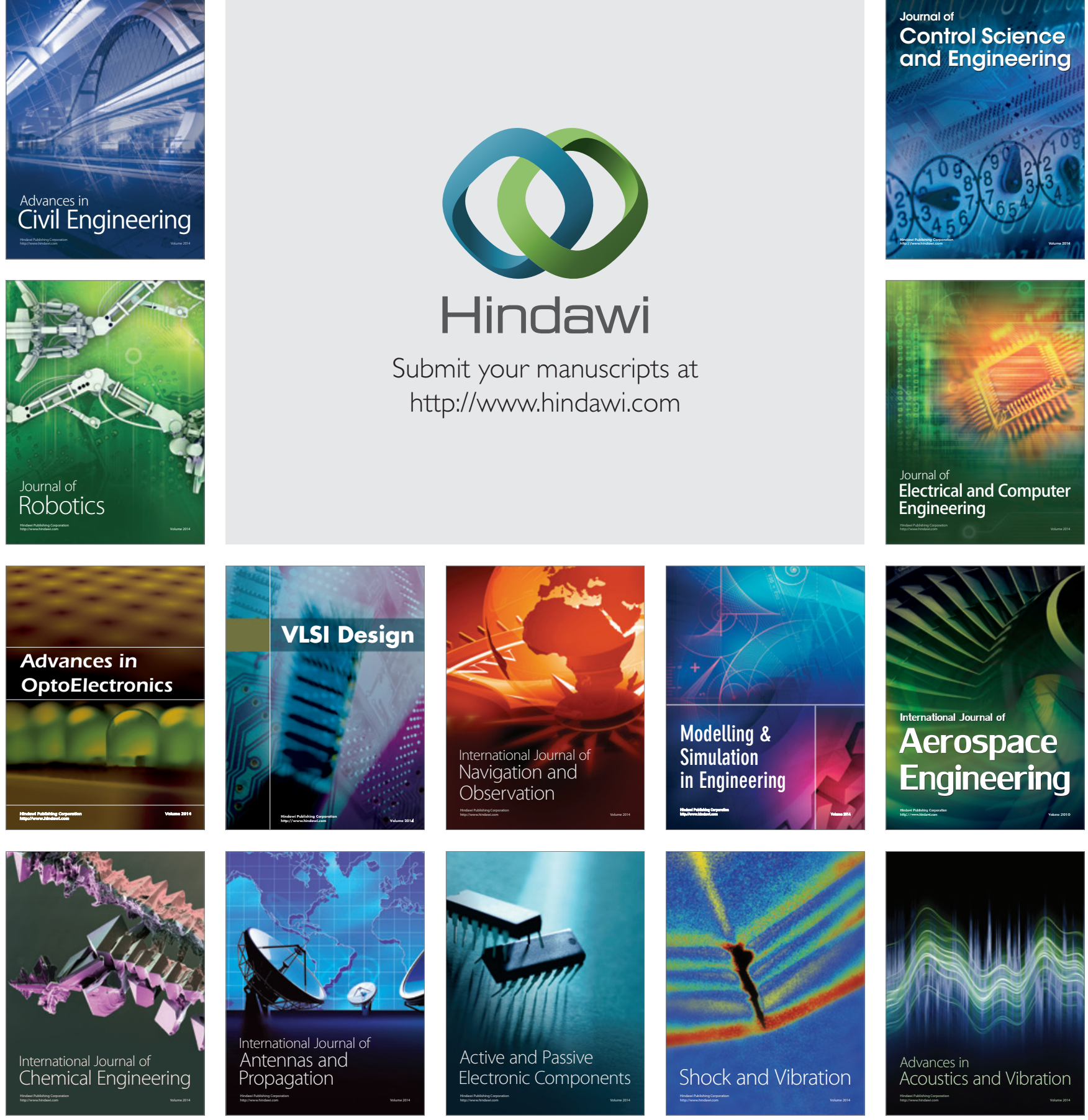